\title{
SINERGIA
}

REVISTA DO INSTITUTO DE CIÊNCIAS ECONÔMICAS, ADMINISTRATIVAS E CONTÁBEIS (ICEAC)

\section{ANÁLISE DOS EFEITOS CROWDING-IN E CROWDING-OUT PARA A ECONOMIA BRASILEIRA}

\author{
RODRIGO NOBRE FERNANDEZ \\ CLAUDIO SHIKIDA \\ GABRIELITO RAUTER MENEZES \\ RAFAEL HENRIQUE MARTINS DE ALMEIDA***
}

\section{RESUMO}

O presente trabalho tem como objetivo estimar um modelo econométrico para testar se ocorreu um efeito deslocamento (crowding-out) durante o período de 1995-2014. Para tanto, utilizou-seo método de Pesaran e Shin (1999, 2001),o qual se estima um modelo ARDL (Autoregressive with Distributed Lags),seguido de um teste de limites (bounds test). Essa metodologia é adequada para pequenas amostras e é flexível para lidar com variáveis de diferentes ordens de integração. Os resultados indicam uma relação positiva entre consumo do governo e investimento privado. Alguns dos fatores pelos quais este efeito pode ter ocorridosão o controle de gastos e a redução da máquina pública entre 1995-2000, políticas utilizadas para estabilização, que contaram com a expansão do investimento privado na economia brasileira. Em um segundo momento, o aumento do dispêndio governamental iniciado, principalmente no segundo mandato do governo Lula e seguido nos governos seguintes, associado à execução do cronograma de investimentos do Programa de Aceleração do Crescimento (PAC).

Palavras-Chave: Crowding-in, Crowding-out, Economia Brasileira.

\section{ABSTRACT}

The present work aims to estimate an econometric model to test if there was a crowding-out effect during the period 1995-2014. To achieve this goal, the method of Pesaran and Shin $(1999,2001)$ was used to estimates an Autoregressive with Distributed Lags (ARDL) model followed by a bounds test. This methodology is suitable for small samples and is flexible to handle with variables of different integration orders. The results indicated a positive relationship between government consumption and private investment. Some factors by which this effect occurred were the control of expenditures and the reduction of the public machine between 1995-2000, policies used for stabilization and that counted on the expansion of private investment in Brazilian economy. Secondly, the increase in government expenditures initiated mainly in the second term of the Lula government and followed in the next governments, associated to the implementation of the Growth Acceleration Program (PAC) investment schedule.

Keywords: Crowding-in, Crowding-out, Brazilian Economy.

Recebido em: 09-10-2017 Aceito em: 20-02-2018

\section{INTRODUÇÃO}

No início dos anos 1990, com a abertura econômica e, posteriormente, com a implantação do plano Real, a economia brasileira deparou-se com um conjunto de políticas públicas que visavam ao controle dos gastos e à redução do tamanho do Estado, ao mesmo tempo em que se buscava aumentar a inclusão social. Tais medidas econômicas foram necessárias para o retorno da estabilidade dos preços da economia, bem como para a tentativa da retomada do crescimento econômico,acompanhada de redução da pobreza.

A política de austeridade fiscal foi relaxada, principalmente no segundo mandato do presidente Lula, tendo sequência nos dois mandatos da presidente Dilma, com políticas como, por exemplo, o PAC (programa de aceleração do crescimento), as quais contêm uma série de investimentos que, segundo seus formuladores, objetivavam melhorar diversos aspectos da infraestrutura, tais como os de habitação, saneamento, transporte, energia etc. Para alguns autores, como, por exemplo, Alston et al.(2016), duas causas importantes para este relaxamento teriam sidos a descoberta do pré-sal e a crise de 2008.

Dentro do contexto da gestão da política fiscal, há, essencialmente, dois pontos de vista distintos

\footnotetext{
"Doutor em Economia Aplicada pelo PPGE da UFRGS. Professor do Programa de Mestrado em Economia Aplicada da UFPEL

"Doutor em Economia Aplicada pelo PPGE da UFRGS. Professor do Programa de Mestrado em Economia Aplicada da UFPEL

*** Doutor em Economia Aplicada pelo PPGE da UFRGS

Graduado em Ciências Econômicas pela UFPEL
} 
sobre os efeitos do aumento do gasto público no investimento privado. A visão econômica tradicional destaca que o aumento das despesas do governo reduziria o investimento privado, provocando, assim, o efeito deslocamento ou crowding-out. Em linhas gerais, o maior gasto governamental, seja ele financiado com impostos ou com a dívida pública, aumenta a demanda por bens e serviços, elevando as taxas de juros, o que torna o capital mais caro e encarece o investimento privado. Contudo, esse efeito pode ser complementar (crowding-in) quando os recursos da economia estão ociosos ou mal-empregados. Um exemplo disso são como as despesas públicas em infraestrutura que podem aumentar o investimento privado, principalmente em países em desenvolvimento (AHMED; MILLER, 2000).

Sob esse prisma, esse trabalho tem por objetivo analisar se ocorreu a complementariedade ou substituição de gastos públicos por privados, tendo como base o período pós-estabilização da economia brasileira, para dados trimestrais de 1995 a 2014.Para tanto, utiliza-se o método de Pesaran e Shin (1999, 2001) para estimar-se um modelo ARDL (Autoregressive With Distributed Lags),com um teste de limites (Bounds Test). Essa metodologia é adequada para pequenas amostras e é flexível para lidar com variáveis de diferentes ordens de integração.

Nesse sentido, as estimações seguem a linha de Jacinto e Ribeiro (1998), visto que as estimativas realizadas nos estudos de Bicudo (2007), dos Santos e Pires (2009) e Sonaglio et al. (2010) são limitadas pelo horizonte temporal referente da variável que serve como medida do investimento privado. Como resultado, encontra-se uma relação positiva entre consumo público e investimento privado no período 19952014, indicando uma possível relação de complementariedade entre essas duas variáveis.

Desta forma, esse trabalho está estruturado em quatro seções, tendo por início essa introdução. $\mathrm{Na}$ segunda parte, e faz-se a literatura. Segue-se pela apresentação da metodologia e dos dados. Posteriormente, mostram-se os resultados e encerram-se com as considerações finais.

\section{REVISÃO DA LITERATURA}

O marco inicial para o estudo da relação entre o investimento privado e os gastos governamentais é o trabalho de Aschauer (1989), o qual aborda que um aumento nas despesas públicas reduz o investimento privado. Contudo, o autor relata que o investimento do setor público em infraestrutura pode ser benéfico para o crescimento do investimento privado, e, nesse caso, ambos canais podem ser complementares.

Como a primeira referência nacional nessa temática, Studart (1992) utilizou dados anuais para o período 1973-1989 e encontrou que, além do efeito atribuído pelo aumento dos gastos do governo, a taxa de inflação seria um dos principais fatores que geraria incerteza para os agentes econômicos afetando, assim, o investimento privado de forma negativa.

Em trabalho posterior, Jacinto e Ribeiro (1998) destacam que Studart (1992) verificou a possibilidade de existência de raízes unitárias nas séries utilizadas. Além disso, os autores postularam que o aumento das emissões de títulos e/ou o aumento da taxa de juros básica por parte do setor público poderia estar associado a uma redução do investimento privado, uma vez que o governo "drena" capital financeiro que, de outro modo, estaria disponível ao financiamento do investimento do setor privado, resultando em crowding-out. Usando os mesmos dados de Studart (1992), mas acrescentando testes de raiz unitária, verificou-se a manutenção da evidência de crowding-out, embora o valor das elasticidades estimadas tenha sido menor.

Nesse mesmo prisma, Melo e Rodrigues Júnior (1999) utilizaram dados anuais para o período 19701995 e encontraram que as políticas elaboradas pelo setor público poderiam, no caso brasileiro, estimular o investimento privado, principalmente se houvesse a fixação da taxa básica de juros em nível moderado e a manutenção da taxa de inflação em níveis baixos. A conjugação de tais fatores poderia, na visão destes autores, reduzir o nível de incerteza para os agentes privados e aumentar a credibilidade dos mesmos acerca da sintonia entre política monetária e fiscal.

Ahmed e Miller (2000)examinaram os efeitos das despesas públicas desagregadas no investimento privado, usando métodos dedados em painel no período de 1975-1984 para 39 países. Em termos gerais, as despesas governamentais financiadas pelo aumento de impostos reduzem maiso investimento privado do que os gastos do governofinanciados pela dívida pública. As despesas de seguridade social reduzem o investimento privado, enquantoas despesas com transporte e comunicação induzem ingressos privados em países em desenvolvimento.

Ferreira (2006), analisando o período 1970-2003, observou que os investimentos públicos em infraestrutura poderiam estimular os investimentos privados. Além disso, e, de modo inverso, os investimentos públicos que não se dirigem a projetos de infraestrutura acabariam por deslocar os investimentos privados, gerando o crowding-out.

Utilizando a metodologia do SVAR (Structural Vector Autoregression),Mitra (2006) encontrou evidências de efeito crowding-out para o caso indiano. Em suma, no curto prazo, o investimento do governo repele o investimento privado. Segundo a autora, isso pode ter ocorrido, porque a Índia teve um ritmo de 
crescimento econômico acelerado com uma menor proporção de investimento público comparada ao privado. Para o caso brasileiro, Mendonça et al (2009), usando dados trimestrais (1995.I - 2007.IV), encontraram evidências de que a resposta contemporânea do aumento do consumo privado em relação aos gastos do governo seria positiva, o que poderia indicar a incidência de crowding-out. Os autores afirmaram que o PIB teria uma probabilidade de $77,1 \%$ de decréscimo após o choque positivo nos gastos públicos e a taxa de juros aumenta com probabilidade de $90 \%$.

Usando um modelo VAR (Vetor Autoregressivo) padrão, Afonso e Aubyn (2009) investigaram a existência dos efeitos crowding in/out para 14 países da EU (União Européia), além de Canadá, Japão e EUA, entre 1960 e 2005.As estimativas dos autores indicaram que o aumento no investimento público acarretou numa contração na produção em cinco casos (Bélgica, Irlanda, Canadá, Reino Unido e Países Baixos). Por outro lado, o efeito de crowding-inprevaleceu em oito casos (Áustria, Alemanha, Dinamarca, Finlândia, Grécia, Portugal, Espanha e Suécia).

Bicudo (2007), analisando o período 1995-2006, constatou que o investimento público, quando financiado por déficit fiscal e taxa de juros elevada, reduziria o volume de crédito disponível para o setor privado, inibindo o investimento (ou seja, encontraram evidências de crowding-out). Luporini e Alves (2010) analisaram os determinantes do investimento privado no Brasil com dados anuais no período 1970-2005, encontrando evidências de que reduções no nível de crédito e as instabilidades políticas no país resultariam em diminuição no nível de investimento privado na economia. Outro resultado é que políticas públicas voltadas àestabilização econômica e àcredibilidade dos agentes econômicos afetariam positivamente o nível de investimentos.

Utilizando o Modelo de Correção de Erro Vetorial (VECM), Sonaglio et al. (2010) observaram o efeito de crowding-out para investimentos públicos e privados na economia brasileira, com dados anuais de variáveis relacionadas à tributação e à taxa de juros para o período 1995-2006. Os resultados evidenciam que o nível de investimento privado estaria intimamente ligado ao comportamento dos investidores frente a mudanças no incentivo fiscal, preço de bens de capitais, e à interação entre os investimentos públicos e privados $^{1}$

Com o uso de dados em painel, Furceri e Souza (2011) encontraram que os gastos do governo produzem importantes efeitos de exclusão (crowding out), afetando negativamente tanto o consumo privado quanto 0 investimento. Os pesquisadores também encontraram possíveis assimetrias do efeito do consumo governamental no consumo e no investimento privado. Em particular, esse efeito varia substancialmente entre as regiões, mas não parece depender da fase do ciclo econômico. Com uma metodologia similar, mas utilizando conjuntamente técnicas de séries temporais, Dreger e Hans-Eggert (2016)exploram a relação de longo prazo entre investimentos públicos e privados na área do euro. Em termos gerais, a falta de investimento público pode ter restringido o investimento privado e, portanto, o crescimento do PIB na área do euro.

Em linhas gerais, grande parte dos trabalhos revisados nesse estudo encontraram uma relação negativa entre o gasto público e o investimento privado. $O$ Quadro 1, a seguir, resume as principais informações apresentadas nas obras destacadas nessa seção.

\begin{tabular}{cccc} 
QUADRO 1 - Resumo das Referências & & \\
\hline Referência & Metodologia & País(es) & Efeito Crowding-Out $^{\star}$ \\
\hline Aschauer (1989) & Séries Temporais & Estados Unidos \\
Studart (1992) & Séries Temporais & Brasil & Sim \\
Jacinto e Ribeiro (1998) & Séries Temporais & Brasil & Sim \\
Melo e Rodrigues Junior (1999) & Séries Temporais & Brasil & Sim \\
Ahmed e Miller (2000) & Dados em Painel & Grupo de Países & Sim \\
Ferreira (2006) & Séries Temporais & Brasil & Sim \\
Mitra (2006) & SVAR & Índia & Sim \\
Bicudo (2007) & Séries Temporais & Brasil & Sim \\
Mendonça et al. (2009) & SVAR & Brasil & Sim \\
Afonso e Aubyn (2009) & VAR & União Européia, Canadá, Estados Unidos e Japão & Sim \\
Luporini e Alves (2010) & VECM & Brasil & Sim \\
Sonaglio et al. (2010) & VECM & Brasil & Sim \\
Furceri e Souza (2011) & Dados em Painel & Grupo de Países & Sim \\
Dreger e Hans-Eggert (2016) & Dados em Painel & União Europeia & Não \\
Soave et al. (2016) & Dados em Painel & Grupo de Países & Não
\end{tabular}

FONTE: Elaborado pelos autores.

NOTA: A última coluna retrata se os autores encontraram alguma evidência do efeito crowding-out nas estimativas.

\footnotetext{
${ }^{1}$ Em estudo indiretamente relacionado ao tema, Soave et al. (2016) analisam um painel $(n=48)$ de países e encontram que o gasto do governo tem efeito crowding-in sobre o consumo privado no longo prazo, com efeitos maiores nos países em desenvolvimento do que nos desenvolvidos.
} 
Por fim, é importante frisar que autores como Aschauer (1989) e Ferreira (2006) destacam que a complementariedade entre os gastos públicos e o investimento privado ocorre principalmente quando esse gasto se refere à infraestrutura. As melhorias ocorridas nesse âmbito podem promover e estimular potencialmente o investimento privado. De modo geral, o efeito crowding-out se mostra predominante no curto prazo, como reforçamMitra (2006), Sonaglio et al. (2010) e Dreger e Hans-Eggert (2016).

\section{DADOS E METODOLOGIA}

A descrição metodológica deste trabalho é feita em duas subseções. Inicialmente,apresenta-se a base de dados e, na sequência, o modelo econométrico utilizado para a análise dos efeitos crowding-in e crowding-out.

\subsection{Dados}

Os dados utilizados neste trabalho são séries de tempo trimestrais no intervalo de 1995.I - 2014.III, coletados a partir do Instituto de Pesquisa Econômica Aplicada (IPEA) e,também, pelo Banco Central do Brasil (BACEN). As variáveis utilizadas, excluindo-se os índices, estão precificadas em milhões de Reais avaliadas a preços do ano 2000. Abaixo, faz-se um breve sumário das mesmas:

Formação bruta de capital fixo (FBCF): Mede o quanto as empresas aumentaram seus bens de capital, sendo eles, basicamente máquinas, equipamentos e material de construção. Essa variável indica a capacidade de produção de uma nação e se os empresários estão confiantes em relação ao futuro. Usamos esse indicador como uma medida do investimento privado na economia.

Consumo Final da Administração Pública (CONS_PUB): São as despesas com serviços individuais e coletivos prestados gratuitamente, total ou parcialmente, pelas três esferas de governo (federal, estadual e municipal), deduzindo-se os pagamentos parciais (entradas de museus, matrículas etc) efetuados pelas famílias. São valorados ao custo de sua produção. Esse indicador é uma medida para os gastos do governo na economia.

Desembolsos do Banco Nacional de Desenvolvimento Econômico e Social (bndes): Mede o total de desembolsos em milhões de Reais concedidos pelo BNDES.

Índice de Geral de Preços (IGPM): O IGPM teve seu surgimento no final da década de 1940 para ser uma medida mais abrangente da verificação do movimento dos preços. O IGP é a média aritmética ponderada de três outros índices de preços, sendo eles: índice de Preços ao Produtor Amplo (IPA), Índice de Preços ao Consumidor (IPC) e Índice Nacional de Custo da Construção (INCC). A legenda M indica que o índice é calculado entre os dias 20 do mês um e 21 do mês dois.

Utilização da Capacidade Instalada (UTCAP): Pode ser definido como o limite ou capacidade de produção industrial. É a quantidade de produto que as máquinas e equipamentos instalados são capazes de produzirem.

\subsection{Modelo Econométrico}

O modelo básico deste artigo baseia-se em Jacinto e Ribeiro (1998), que, numa especificação linear, assume a seguinte forma:

$$
\mathrm{FBCF}=\alpha+\beta_{1} \mathrm{FBCF}_{t-1}+\beta_{2} \mathrm{CONS} \_\mathrm{PUB}+\beta_{3} \mathrm{BNDES}+\beta_{4} \mathrm{UTCAP}+\beta_{5} \mathrm{IGPM}+\varepsilon_{t}
$$

sendo: $\mathrm{FBCF}=$ investimento privado; $C O N S \_P U B=$ investimento público; BNDES $=$ crédito fornecido pelo BNDES; UTCAP = utilização da capacidade instalada e IGPM = taxa de inflação (os autores a consideraram como uma variável de incerteza em relação ao futuro). Tem-se que $\varepsilon_{t}$ é um distúrbio aleatório. A teoria econômica sugere que $\beta_{1}>0, \beta_{2}>0$ ou $\beta_{2}<0, \beta_{3}>0, \beta_{4}>0$ e $\beta_{5}<0$.

Aespecificação acima pode apresentar problemas por dois motivos: (a) a especificação ad hoc que impõe que apenas uma variável tenha defasagens (e apenas uma defasagem) e, (b) o fato de que a ordem de integração das variáveis seja distinta. Além disso, a base de dados tem reduzido número de observações, o que não é apropriado para uma análise de cointegração à la Johansen (1995), por exemplo.

Desta forma, neste trabalho, utilizou-se a metodologia de Pesaran e Shin $(1999,2001)$, que envolve a estimação de um modelo ARDL com testes de limites (bounds test). Esta metodologia tem a vantagem de ser adequada para a estimação de relações de cointegração em pequenas amostras, como sugerem Ghatak e Siddiki (2001). Tal metodologia não sofre da limitação da estimação ad hoc (pode-se estimar tantos ARDLs quantas sejam as variáveis do modelo) além de ser flexível o suficiente para acomodar 
variáveis com diferentes ordens de integração $(I(0) \text { e } I(1))^{2}$.

O procedimento de Pesaran e Shin $(1999,2001)$ permite verificar a existência de cointegração entre as variáveis, mesmo que elas não tenham o mesmo número de raízes unitárias ${ }^{3}$, a partir da estimação da seguinte equação:

$$
\Delta y_{t}=-\sum_{i=1}^{p-1} \gamma_{i}^{*} \Delta y_{t-1}+\sum_{j=1}^{k} \sum_{i=0}^{q_{j}-1} \Delta x_{j, t-1}{ }^{\prime} \beta_{j, i} *-\rho y_{t-1}-c-\sum_{j=1}^{k} x_{j, t-1}{ }^{\prime} \delta_{j}+\varepsilon_{t}
$$

Da qual se testa a hipótese nula de não-existência de cointegração:

$$
\begin{aligned}
& \rho=0 \\
& \delta_{1}=\ldots=\delta_{k}=0
\end{aligned}
$$

Este é o chamado bounds test de Pesaran e Shin. Para o caso de variáveis com graus de integração diferente, utilizam-se os valores críticos tabulados por Pesaran, Shin e Smith (2001). Vale ressaltar que as especificações acima podem incluir termos deterministas como dummies sazonais e tendência deterministas, o que possibilita controlar, por especificidades das séries de tempo, ainda que em amostras reduzidas.

\section{RESULTADOS}

Inicialmente, apresentamos as estatísticas descritivas das variáveis utilizadas.

TABELA 1 - Estatísticas Descritivas das Variáveis

\begin{tabular}{cccccc}
\hline & FBCF & CONS_PUBLICO & UTCAP & BNDES & IGPM \\
\hline Média & 104066.7 & 121510.0 & 80.89722 & 149.8100 & 2.078608 \\
Máximo & 231331.7 & 333384.5 & 84.73000 & 1323.718 & 12.81000 \\
Mínimo & 31363.06 & 35635.71 & 75.58000 & 7.660800 & -1.52000 \\
Desvio Padrão & 65427.10 & 76998.33 & 2.085277 & 236.9987 & 2.126537 \\
Número de & 79 & 79 & 79 & 79 & 79 \\
Observações & & & &
\end{tabular}

FONTE: Elaborado pelos autores.

Vale observar que o maior valor da variável CONS PUBLICO foi em 2013 e o menor valor em 1995, com 333,4 e 35,6 bilhões de reais respectivamente. Os valores máximos e mínimos FBCF também foram registrados, respectivamente, no ano de 2013, com um montante de 231,3 bilhões de reais, e, em 1995, com 31,4 bilhões de Reais.

Verifica-se a existência de possível padrão sazonal nas séries. Ao invés de se dessazonalizar as séries, contudo, optou-se por modelar a sazonalidade com a inclusão de dummies no ARDL ${ }^{4}$. Ademais, segue-se a estimação de Jacinto e Ribeiro (1998), isto é, todas as variáveis, exceto o IGPM, foram logaritmizadas.

O primeiro passo do teste de cointegração consiste em verificar o grau de integração de cada variável. Inicialmente, apresentam-se os resultados para os testes de Dickey e Fuller, acrescentado do teste de Phillips e Perron (PP). Os testes foram realizados em nível e em primeira diferença, e se encontram na Tabela 2 a seguir.

\footnotetext{
${ }^{2}$ Pahlavani, Wilson e Worthington (2005) e Belloumi (2013) apontam também outra vantagem do método: evitar a complexidade de escolhas envolvendo a inclusão dos termos deterministas, típico de estimações que utilizam a metodologia de Johansen.

${ }^{3}$ Desde que não exista alguma variável I(2) no modelo.

${ }^{4} \mathrm{~A}$ escolha pelo uso de dummies sazonais deveu-se à pequena extensão das séries $(\mathrm{n}=79)$. Mesmo sem se considerar o reduzido número de observações das séries utilizadas, sabe-se que, conforme Enders (2010), processos de dessazonalização nem sempre captam perfeitamente os padrões sazonais.
} 
TABELA 2 - Teste Dickey-Fuller (ADF) e Philips-Perron (PP)

\begin{tabular}{cccccc}
\hline Critérios & Variáveis & \multicolumn{2}{c}{ Em Nível } & \multicolumn{2}{c}{ Em Diferença } \\
\hline ADF & & Estatística t & p-valor & Estatística t & p-valor \\
PP & LFBCF & -0.450486 & 0.8941 & -10.88614 & 0.0001 \\
ADF & LFBCF & -0.353315 & 0.9110 & -8.952345 & 0.0000 \\
PP & LCONS_PUBLICO & 0.218590 & 0.9721 & -5.438366 & 0.0000 \\
ADF & LCONS_PUBLICO & -0.684529 & 0.8439 & -42.40405 & 0.0001 \\
PP & LUTCAP & -1.546205 & 0.5047 & -5.853238 & 0.0000 \\
ADF & LUTCAP & -2.846700 & 0.0565 & -15.30849 & 0.0001 \\
PP & LBNDES & -1.338205 & 0.6078 & -17.20613 & 0.0001 \\
ADF & LBNDES & -8.347007 & 0.0000 & -39.95598 & 0.0001 \\
PP & IGPM & -5.520736 & 0.0000 & -9.258482 & 0.0000 \\
\hline
\end{tabular}

FONTE: Elaborado pelos autores.

Verifica-se que todas as séries são estacionárias na primeira diferença, com exceção do IGPM. Entretanto, como indica Perron (1989), quando as séries apresentam quebras estruturais, ao serem submetidas ao teste de raiz unitária as mesmas podem apresentar um falso positivo. Desse modo, foram também realizados testes de raiz unitária com quebra estrutural. Sob a hipótese nula da existência de raiz unitária, foram obtidos os seguintes resultados expostos na Tabela 3:

TABELA 3 - Teste de Raiz Unitária com Quebra

\begin{tabular}{ccccccc}
\hline & \multicolumn{3}{c}{ Em Nível } & & \multicolumn{2}{c}{ Em Diferença } \\
\hline Variável & Estatística t & p-valor & $\begin{array}{c}\text { Data de } \\
\text { Quebra }\end{array}$ & Estatística t & $\begin{array}{c}\text { Data de } \\
\text { Q-valor }\end{array}$ \\
\hline LFBCF & -2.192800 & 0.9637 & $2003 Q 04$ & -11.47726 & $<0.01$ & $2008 Q 03$ \\
LCONS_PUBLICO & -2.513670 & 0.8995 & $2001 Q 03$ & -34.60808 & $<0.01$ & $2000 Q 03$ \\
LUTCAP & -5.095122 & $<0.01$ & $2004 Q 01$ & -6.688118 & $<0.01$ & 2009 Q01 \\
LBNDES & -3.382635 & 0.4527 & $2008 Q 02$ & -21.19274 & $<0.01$ & $1999 Q 01$ \\
IGPM & -8.169560 & $<0.01$ & $2002 Q 04$ & -9.869241 & $<0.01$ & $2003 Q 02$ \\
\hline
\end{tabular}

FONTE: Elaborado pelos autores.

O teste indicou que as variáveis FBCF, CONS PUB e BNDES não são estacionárias em nível e, para elas, os possíveis pontos de quebra seriam 2003Q4, 2001Q03 e 2008Q2. Destaca-se que, no ano 1999, ocorreu a mudança no regime do câmbio do país, quando foi abandonado o regime de bandas cambiais em favor da flutuação cambial. Já, em 2003, os pontos de quebras podem ser atribuídos às eleições presidenciais de 2002, com a posse do ex-presidente Lula em 2003. Já a crise subprime de 2008 pode ser pensada como uma explicação para último ponto de quebra. Após tomar-se a primeira diferença de todas as séries, exceto o IGPM, todas se tornaram estacionárias, sendo os possíveis pontos de quebra entre os anos 1999-2000 e 2008-2009. Desta forma, optamos pela inclusão de duas dummies de quebra no modelo.

A fim de testar a existência de cointegração entre as variáveis, optou-se por estimar cinco modelos ARDL, alterando a variável dependente do modelo. Como o número de observações é pequeno $(n=79)$, o número de defasagens foi especificado a partir de um número máximo igual a cinco. Quanto aos termos deterministas, além das dummies de quebra, foram incluídas três dummies trimestrais.Os resultados são expostos na Tabela 4 a seguir

TABELA 4 - Resumo dos Resultados

\begin{tabular}{|c|c|c|c|c|}
\hline Variável Dependente & $\begin{array}{c}\text { Número de } \\
\text { defasagens (critério } \\
\text { AIC) }\end{array}$ & $\begin{array}{c}\text { Teste de } \\
\text { Autocorrelação } \\
\text { dos resíduos } \\
\text { (Breusch-Godfrey, } \\
\mathrm{k}=4 \text { ) }\end{array}$ & $\begin{array}{c}\text { Estatística F } \\
\text { (limites inferior e } \\
\text { superior) }\end{array}$ & $\begin{array}{c}\text { H0: Não existe relação } \\
\text { de cointegração }\end{array}$ \\
\hline LFBCF & $\operatorname{ARDL}(4,0,5,4,0)$ & $\begin{array}{l}F=2.11, \quad(p \text {-valor: } \\
0.09)\end{array}$ & $0.42(3.07-4.44)$ & Não há \\
\hline LCONS_PUBLICO & $\operatorname{ARDL}(5,5,3,4,2)$ & $0.19(0.94)$ & $2.91(3.07-4.44)$ & Não há \\
\hline LUT̄CAP & $\operatorname{ARDL}(2,5,2,5,0)$ & $2.57(0.05)$ & 0.31 & Não há \\
\hline LBNDES & $\operatorname{ARDL}(1,0,5,0,0)$ & $0.76(0.55)$ & 16.23 & Há \\
\hline IGPM & $\operatorname{ARDL}(1,5,2,5,3)$ & $0.21(0.93)$ & 6.92 & Há \\
\hline Lower-upper bounds & \multicolumn{4}{|c|}{$3.07-4.44$} \\
\hline
\end{tabular}

FONTE: Elaborado pelos autores.

NOTA: Em todas as especificações, foram incluídas dummies para os três primeiros trimestres do ano e dummies para 1999 e 2008

Das duas relações de cointegração que parecem existir, analisa-sea que expressa uma das variáveis que mede o investimento público (LBNDES) como variável dependente para a análise de cointegração. A forma do VECM é a que se segue. 
TABELA 5 - Estimação da Equação de Cointegração

\begin{tabular}{lrrrr}
\hline Variável & Coeficiente & Erro Padrão & Estatística t & P-Valor \\
\hline D(LFBCF) & 3.121481 & 2.857801 & 1.092267 & 0.2792 \\
D(LCONS_PUBLICO) & 0.082585 & 1.76392 & 0.046819 & 0.9628 \\
D(LCONS_PUBLICO(-1)) & -1.65155 & 1.395273 & -1.18367 & 0.2413 \\
D(LCONS_PUBLICO(-2)) & -0.96454 & 1.168476 & -0.82547 & 0.4124 \\
D(LCONS_PUBLICO(-3)) & 2.373798 & 1.437108 & 1.651788 & 0.1039 \\
D(LCONS_PUBLICO(-4)) & 5.682468 & 1.945388 & 2.920995 & 0.0049 \\
D(LUTCAP) & -9.96965 & 11.41411 & -0.87345 & 0.386 \\
D(IGPM) & 0.034038 & 0.041279 & 0.824575 & 0.4129 \\
D(D1999) & 0.39388 & 0.602478 & 0.653767 & 0.5158 \\
D(D2008) & 0.049388 & 0.538315 & 0.091746 & 0.9272 \\
D(TRIMESTRE1) & 1.029757 & 0.356689 & 2.886986 & 0.0054 \\
D (TRIMESTRE2) & -0.80232 & 0.372669 & -2.15289 & 0.0354 \\
D(TRIMESTRE3) & -0.13768 & 0.315147 & -0.43689 & 0.6638 \\
CointEq(-1) & -0.98253 & 0.105294 & -9.33128 & 0.0000 \\
\hline FONTE: Elaborado Per & & &
\end{tabular}

FONTE: Elaborado pelos autores.

De acordo com os resultados encontrados na tabela 5, observa-se que a velocidade de ajuste é elevada (-0.98, na última linha, segunda coluna), o que é um indício de existência da relação de longo prazo. Aparentemente, as dummies sazonais parecem ter cumprido seu papel de absorver os padrões sazonais das séries. Os coeficientes de longo prazo são apresentados a seguir na Tabela 6.

TABELA 6 - Coeficientes de Longo Prazo

\begin{tabular}{crrrr}
\hline Variável & Coeficiente & Erro Padrão & Estatística t & P-Valor \\
\hline LFBCF & 2.149999 & 1.494971 & 1.438154 & 0.1557 \\
LCONS_PUBLICO & -1.105852 & 1.516739 & -0.729099 & 0.4688 \\
LUTCAP & -1.766729 & 0.454358 & -3.888409 & 0.0003 \\
IGPM & -0.020018 & 0.045417 & -0.440759 & 0.6610 \\
D1999 & 1.162800 & 0.456008 & 2.549958 & 0.0134 \\
D2008 & -0.116954 & 0.420538 & -0.278106 & 0.7819 \\
DTRIMESTRE1 & 1.190643 & 0.528128 & 2.254460 & 0.0279 \\
DTRIMESTRE2 & -0.910465 & 0.558178 & -1.631138 & 0.1082 \\
DTRIMESTRE3 & -0.261727 & 0.491241 & -0.532787 & 0.5962 \\
\hline
\end{tabular}

FONTE: Elaborado pelos autores.

Não foi encontrada significância estatística na relação estimadaentre os investimentos privado e público (medido pela variável dependente LBNDES). Entretanto, a elasticidade de longo prazo da utilização da capacidade instalada estimada é elevada (-1.7667)e é estatisticamente significativa, indicando que aumentos da utilização da capacidade instalada tenderiam a diminuir os desembolsos do BNDES. A quebra da mudança do regime cambial - captada pela variável D1999 - também se mostrou estatisticamente significativa no longo prazo, representado umimpacto positivo da adoção do câmbio flexível sobre os desembolsos do BNDES.

No teste de estabilidade do modelo, contudo, os resultados foram ambíguos. O teste CUSUM sugere instabilidade nos parâmetros estimados, enquanto o CUSUM ao quadrado sugere o oposto.Diante destes resultados ambíguos e dado o reduzido número de observações, optou-se por investigar as relações de causalidade entre as variáveis. Para tanto, utilizou-se a metodologia de Toda e Yamamoto (TODA;YAMAMOTO, 1995).

Neste caso, a melhor especificação - em termos de não-autocorrelação dos resíduos - excluiu as dummies (que não se mostraram estatisticamente significativas) e contou com a inclusão de uma tendência determinista e sete defasagens das variáveis endógenas. Os resultados da estimação desta especificação encontram-se na Tabela 7. 
TABELA 7 - Procedimento de Toda-Yamamoto

\begin{tabular}{|c|c|c|c|c|c|c|}
\hline & \multicolumn{5}{|c|}{$x^{2}$} & \multirow[t]{2}{*}{ Causalidade } \\
\hline Variável dependente & LBNDES & LUTCAP & LFBCF & IGPM & LCONS_PUBLICO & \\
\hline LBNDES & - & 3.11 & 3.87 & $13.34\left(^{*}\right)$ & 5.63 & IGPM $\rightarrow$ LBNDES \\
\hline LUTCAP & 8.67 & - & 9.99 & $16.51\left(^{\star \star}\right)$ & $14.22\left(^{* \star}\right)$ & $\begin{array}{c}\text { LCONS_PUBLICO } \rightarrow \text { LUTCAP, } \\
\text { IGPM } \rightarrow \text { LUTCAP, }\end{array}$ \\
\hline LFBCF & 3.75 & $20.58(* *)$ & - & $12.38\left(^{*}\right)$ & 9.64 & LUTCAP $\rightarrow$ LFBCF, IGPM $\rightarrow$ LFBCF \\
\hline $\begin{array}{c}\text { IGPM } \\
\text { LCONS_PUBLICO }\end{array}$ & $\begin{array}{c}9.03 \\
\left.14.92^{* *}\right)\end{array}$ & $\begin{array}{c}8.34 \\
18.75\left(^{* \star *}\right)\end{array}$ & $\begin{array}{c}8.82 \\
25.16\left(^{* * *}\right)\end{array}$ & $13.34(* *)$ & $\begin{array}{c}13.97\left({ }^{* *}\right) \\
-\end{array}$ & $\begin{array}{l}\text { LCONS_PUBLICO } \rightarrow \text { IGPM } \\
\text { LBNDES } \rightarrow \text { LCONS_PUBLICO, } \\
\text { LUTCAP } \rightarrow \text { LCONS_PUBLICO, } \\
\text { LFBCF } \rightarrow \text { LCONS_PUBLICO, } \\
\text { IGPM } \rightarrow \text { LCONS_PUBLICO }\end{array}$ \\
\hline
\end{tabular}

FONTE: Elaborado pelos autores.

NOTA: $\left(^{*}\right),\left({ }^{* *}\right)$ e $\left({ }^{* *}\right)$, respectivamente, denotam significância a 10,5 e $1 \%$.

Neste caso, a evidência de causalidade é a de que o investimento privado impacta positivamente no consumo público, o que pode ser entendido como uma evidência de complementaridade (crowding-in). Uma forma de se visualizar esta relação é por meio da decomposição da variância do consumo público. Isto é feito na Tabela 8.

TABELA 8 - Decomposição da Variância do Consumo Público

\begin{tabular}{ccccccc}
\hline Período & $\begin{array}{c}\text { Erro } \\
\text { Padrão }\end{array}$ & LUTCAP & LFBCF & IGPM & LBNDES & LCONS_PUBLICO \\
\hline 1 & 0.010667 & 7.077314 & 0.235003 & 10.67542 & 3.803536 & 78.20873 \\
2 & 0.014456 & 6.272258 & 8.884530 & 13.16412 & 3.321795 & 68.35730 \\
3 & 0.016389 & 6.356939 & 10.27972 & 18.69511 & 10.89106 & 53.77717 \\
4 & 0.017638 & 6.844651 & 21.06735 & 16.38006 & 10.31014 & 45.39780 \\
5 & 0.018354 & 8.948739 & 14.38397 & 21.37894 & 7.558389 & 47.72996 \\
6 & 0.019008 & 8.433247 & 21.26686 & 19.29637 & 6.854938 & 44.14859 \\
7 & 0.019583 & 16.41793 & 17.23975 & 21.21709 & 9.089102 & 36.03614 \\
8 & 0.020143 & 19.48645 & 29.16921 & 16.28442 & 6.701141 & 28.35878 \\
9 & 0.020803 & 16.62737 & 25.14020 & 16.92876 & 5.996856 & 35.30682 \\
10 & 0.021396 & 14.61936 & 32.65301 & 14.77307 & 7.184451 & 30.77011 \\
\hline
\end{tabular}

FONTE: Elaborado pelos autores.

Nota-se que a decomposição do erro de previsão do consumo público, ao final de dez períodos, conta com o investimento privado ( $L F B C F)$ em praticamente um terço $(32.65 \%)$. Esta evidência é compatível tanto com a visão de que há uma relação virtuosa entre o setor privado e o setor público, quanto com a visão de que há uma relação de compadrio entre ambos. Durante o período de análise é possível que tenham ocorrido períodos compatíveis com ambas e, portanto, um possível aprofundamento deste trabalho deveria analisar mais cuidadosamente esta relação.

Adicionalmente, percebe-se que as estimativas se encontram alinhadas com os trabalhos de Aschauer (1989),Ferreira (2006), Afonso e Aubyn (2009) e Dreger e Hans-Eggert (2016), no sentido que o gasto público pode ser complementar ao investimento privado, principalmente se o mesmo for destinado a projetos de infraestrutura. Ademais, a relação entre o aumento do gasto público e a redução do investimento privado (crowding-out) pode ser vista principalmente no curto prazo, como é destacado por Jacinto e Ribeiro (1998), Mendonça et al (2009) e Sonaglio et al. (2010).

\section{CONSIDERAÇÕES FINAIS}

Há um debate recorrente entre os formuladores de políticas públicas sobre se o aumento do gasto público reduz o investimento privado ou se Ihe é complementar. Em linhas gerais, as evidências sobre o tema indicam que os efeitos de curto prazo implicam substituição entre essas duas variáveis macroeconômicas (crowding-out). Já, no longo prazo, pode haver a complementariedade entre os investimentos público e o privado (crowding-in).

Este trabalho buscou responder à seguinte pergunta para a economia brasileira: houve crowdingout/crowding-in no decorrer do período de 1995 a 2014? A evidência encontrada é a de uma relação positiva entre o consumo público e o investimento privado indicando uma possível complementariedade (crowding-in) entre essas duas variáveis.

Alguns dos fatores pelos quais este efeito pode ter ocorridosão o controle de gastos e a redução da máquina pública entre 1995-2000, políticas utilizadas para estabilização, que contaram com a expansão do investimento privado na economia brasileira.

Em um segundo momento, o aumento do dispêndio governamental iniciado, principalmente no segundo mandato do governo Lula e seguido nos governos seguintes, associado à execução do cronograma 
de investimentos do Programa de Aceleração do Crescimento (PAC) e ao fato de que os efeitos da crise financeira global na economia brasileira - sobretudo no último trimestre de 2008 e no primeiro trimestre de 2010 - aumentou a demanda por ações estabilizadoras de política fiscal.

Portanto, mesmo considerando o aumento expressivo das captações de recursos pela emissão de ações e do aumento da liquidez internacional articulada como a melhora do risco soberano e do risco das grandes empresas brasileiras, reduzindo a escassez de fundos emprestáveis para as mesmas, a maior participação do governo como absorvedor de poupança doméstica e externa fez com que a parcela do investimento privado não tenha aumentado. Talvez essa seja a explicação da relação de complementaridade encontrada neste trabalho entre o aumento do consumo público e o investimento privado.

Finalmente, para uma nova agenda de pesquisa, é interessante que sejam analisados modelos econométricos que permitam verificar possíveis mudanças de regime, por exemplo, no sentido de Markov. Outra possibilidade seria o uso de séries temporais mistas.

\section{REFERÊNCIAS}

AFONSO, A.; AUBYN, M. Macroeconomic Rates of Return of Public and Private Investment: Crowding-in and Crowdingout Effects. The Manchester School Suplement, 2009.

AHMED, H.; MILLER, S. Crowding-out and Crowding-in effects of the components of government expenditure. Contemporary Economic Policy, 2000.

ALSTON, L.; MELO, M.A.; MUELLER, B.; PEREIRA, C. Brazil in Transition: Beliefs, Leadership, and Institutional Change. Princeton, Princeton University Press, 2016, 280p.

ASCHAUER, D. A. Does Public Capital Crowd Out Private Capital? Journal of Monetary Economics, v.24 pp.171-188. 1989.

BELLOUMI, M. The relationship between trade, FDI and economic growth in Tunisia: an application of the autoregressive distributed lag model. Economic Systems, v.38, n.2, p.269-287, 2013.

BICUDO, R. Uma análise do relacionamento entre investimento público e privado no Brasil. Dissertação (Mestrado em Economia) - Instituto de Ensino e Pesquisa - São Paulo, SP, 2007.

DOS SANTOS, C. H ; PIRES, M. C. C. . Qual a sensibilidade dos investimentos privados a aumentos na carga tributária brasileira? Uma investigação econométrica. Revista de Economia Política, v. 29, p. 1-1, 2009.

DREGER, C.; HANS-EGGERT, R. Does Public Investment Stimulate Private Investment? Evidence for the Euro Area. Economic Modelling, 2016.

ENDERS, W. 2010. Applied econometric time series. New York, John Wiley and Sons, $517 \mathrm{p}$.

FERREIRA, G. M. J. Evolução dos Investimentos no Brasil: Uma Análise Econométrica. Dissertação (Mestrado em Economia de Empresas) - Fundação Getulio Vargas - SP, 2006.

FURCERI, D. SOUSA, R. The Impact of Government Spending on the Private Sector: Crowding-out versus Crowding-in Effects. KYKLOS, Vol. 64, 2011.

JACINTO, P. A.; RIBEIRO, E. P. Cointegração, efeitos crowding-in e crowding-out entre investimento público e privado no Brasil: 1973-89. Teoria e Evidência Econômica, Passo Fundo, v. 5, p. 143-158, 1998.

JOHANSEN, S. Likelihood-Based Inference in Cointegrated Vector Autoregressive Models. Oxford University Press. Oxford, 1995.

LUPORINI, V.; ALVES, J. Investimento Privado: Uma Análise Empírica para o Brasil. Campinas, Economia e Sociedade, v.9, n.3, 2010.

MELO, G. M.; RODRIGUES JÚNIOR, W. Determinantes do Investimento Privado no Brasil: 1970-1995. Brasília, Texto de Discussão IPEA 605, 1998.

MENDONÇA, M. J. C. et al. Avaliando os Efeitos da Política Fiscal no Brasil: resultados de um procedimento de identificação agnóstica 2008 (Texto para discussão IPEA 1377) 2008.

MITRA, P. Has Government Investment Crowded out Private Investment in India? The American Economic Review, Vol. 96, No. 2, pp. 337-341, 2006.

PAHLAVANI, M.; WILSON, E.; WORTHINGTON, A.C. Trade-GDP Nexus in Iran: An Application of the Autoregressive Distributed Lag (ARDL) Model. American Journal of Applied Sciences, vol2, n.7, pp.1158-1165.

PERRON, P. The Great Crash, the Oil Price Schock, and the Unit Root Hypothesis. Econometrica, 1989.

PESARAN, M.H.; SHIN, Y. An Autoregressive Distributed Lag Modeling Approach to Cointegration Analysis. In: STROM, S. (ed). Econometrics and Economic Theory in the $20^{\text {th }}$ Century: The Ragnar Frisch centennial Symposium. Cambridge University Press, Cambridge, 1999.

PESARAN, M.H.; SHIN, Y.; SMITH, R. Bounds testing approaches to the analysis of level relationships. Journal of Applied Econometrics v.16, pp.289-326, 2001. 
PESARAN, M.H.; SMITH, R. Structural Analysis of cointegration VARs. Journal of Economic Survey, v.12, pp.471-505, 1998.

SONAGLIO, C. M.; BRAGA, M. J.; CAMPOS A. C. Investimento Público e Privado no Brasil: Evidências de Crowding-in e Crowding-out no Período 1995-2006. Brasília, EconomiA, v.11, n.2, 2010.

SOAVE, G. P.; AUGUSTO, F.; GOMES, R.; SAKURAI, S. N. Efeitos do gasto do governo sobre o consumo privado: Evidências de países desenvolvidos e em desenvolvimento. Estudos Econômicos (São Paulo), v. 46, p. 5-41, 2016.

STUDART, G. G. Investimentos públicos e privados no Brasil: análise empírica da relação de curto e longo prazos durante o período 1972-1989. In XXII Encontro Nacional de Economia, 1992, Campos de Jordão, Anais, 1992.

TODA, H.; YAMAMOTO, T. Statistical inference in vector autoregression with possibly integrated processes. Journal of Econometrics, v.66, p.225-50, 1995. 